suggests developed aquatic habits; as above noted, some of this family acquired large brains, and persisted late into the Miocene. A still more remarkable likeness to the cats is exhibited in the Palæonictis family, which, unlike the Hyænodons, forms its sectorials out of exactly the same teeth as the true cats. The first American Palæonictis was found two years ago by Wortman, and this author and myself have suggested that this may be the long-sought ancestor of the Felidæ. The Civets are anticipated in the Proviverridæ; yet both Cope and Scott, the highest authorities on this subject, believe that the dog-like Miacidæ alone formed the connecting link between the Creodonta and the true Carnivora.

The foot structure of the ancient Puerco ungulates is stil only partly known. Cope has divided these animals into the Amblypoda and Condylarthra. The Amblypoda are represented in the Puerco by a large form called Pantolambda, with selenodont triangular upper molars, and possibly by Periptychus, with bunodont triangular molars. The Pantolambda molars were, as Cope has shown, converted into those of Coryphodon, the great lophodont Amblypod of the Wahsatch, by a process exactly analogous to that in which the anterior half of a Palæotherium molar was formed, that is, they acquired outer and anterior crests but no posterior crests. This Coryphodon molar type was still later converted into the Uintatherium type by swinging around the outer crest into a transverse crest. I have recently made a careful study of the fore and hind feet of Coryphodon, and have found that while the fore-foot was subdigitigrade like that of the elephant, the hind-foot was fully plantigrade, the entire sole resting upon the ground. The relation or connection between the Bridge Dinocerata and these earlier Amblypoda is still unknown. The Puerco Periptychus left no descendants. The other ungulates of the Puerco were the Condylarthra, the primitive Phenacodontidæ, the supposed ancestors of the Artiodactyls and Perissodactyls. Much remains to be done to clear up this question.

Thus an immense number of problems still await solution, and demand the generous co-operation of European and American specialists in the use of similar methods of research, in the prompt publication of descriptions and figures, and in the free use of museum collections. I may be pardoned for calling general attention to the service which the palæontological department of the American Museum is trying to render in the immediate publication of stratigraphical and descriptive tables of western horizons and localities.

\section{The Factors of Evolution.}

A few words in conclusion upon the impressions which a study of the rise of the mammalia gives as to the factors of organic evolution. I refer also to recent papers by Cope, Scott, and myself.

The evolution of a family like the Titanotheres presents an uninterrupted march in one direction. While apparently prosperous and attaining a great size, it was really passing into a great corral of inadaptation to the grasses which were introduced in the Middle Miocene. So with other families and lesser lines extinction came in at the end of a term of development and high specialisation. With other families no cause for extinction can be assigned, as in the lopping off of the smaller Miocene perissodactyls. The point is that a certain trend of development is taken leading to an adaptive or inadaptative final issue--but extinction or survival of the fittest seems to exert little influence en route.

The changes en route lead us to believe either in predestination-a kind of internal perfecting tendency-or in kinetogenesis. For the trend of evolution is not the happy resultant of many trials, but is heralded in structures of the same form all the world over and in age after age, by similar minute changes advancing irresistibly from inutility to utility. It is an absolutely definite and lawful progression. The infinite number of contemporary developing, degenerating, and stationary characters preclude the possibility of fortuity. There is some law introducing and regulating each of these variations, as in the variations of individual growth.

The limits of variation seem to lie partly in what I have called the "potential of evolution." As the oösperm or fertilised ovum is the potential adult, so the Eocene molar is the potential Miocene molar. We have seen that the variations of the horse and rhinoceros molars, apparently so diverse, are really uniform--is not this evidence that the perissodactyl stem No. 1263 , VOL. 49$]$ had these variations in potentia, waiting to be called forth by certain stimuli? This capacity of similar development unde certain stimuli is part of the law of mammalian evolution, but this does not decide the crucial point whether the reaction is spontaneous in the germ or inherited from the parent. I incline to the latter opinion.

H. F. OSBORN.

\section{A DYNAMICAL THEORY OF THE ELECTRIC AND LUMINIFEROUS MEDIUM.'}

I $\mathrm{T}$ is only at the end of the last century that the somewhat vague principle of the economy of action or effort in physical actions-which, like all other general principles in the scientific explanation of nature, is ultimately traceable to a kind of metaphysical origin-has culminated in the hands of Lagrange in his magnificent mathematical generalisation of the dynamical laws of material systems. Before the date of this concise and all-embracing formulation of the laws of dynamics there was not available any engine of sufficient power and generality to allow of a thorough and exact exploration of the properties of an ultimate medium, of which the mechanism and mode of action are almost wholly concealed from view. The precise force of Lagrange's method, in its physical application, consists in its allowing us to ignore or leave out of account altogether the details of the mecharism, whatever it is, that is in operation in the phenomena under discussion ; it makes everything depend on a single analytical function representing the distribution of energy in the medium in terms of suitable co-ordinates of position and of their velocities ; from the location of this energy, its subsequent play and the dynamical phenomena involved in it are all deducible by straightforward mathematical analysis.

The problem of the correlation of the physical forces is thus divisible into two parts, (i.) the determination of the analytical function which represents the distribution of energy in the primordial medium.which is assumed to be the ultimate seat of all phenomena; and (ii.) the discussion of what properties may be most conveniently and simply assigned to this medium, in order to describe the play of energy in it most vividly, in terms of the stock of notions which we have derived from the observation of that part of the interaction of natural forces which presents itself directly to our senses, and is formulated under the name of natural law. It may be held that the first part really involves in itself the solution of the whole problem; that the second part is rather of the nature of illustration and explanation, by comparison of the intangible primordial medium with other dynamical systems of which we can directly observe the phenomena.

The chief representative of exact physical speculation of the second of these types has been Lord Kelvin. In the older attempts of this kind the dynamical basis of theories of the constitution of the æther consisted usually in a play of forces, acting at a distance, between ultimate elements or molecules of the medium ; from this we must, however, except the speculations of Greek philosophy and the continuous vortical theories of the school of Descartes, which were of necessity purely descriptive and imaginative, not built in a connected manner on any rational foundation. It has been in particular the aim of Lord Kelvin to deduce material phenomena from the play of inertia involved in the motion of a structureless primordial fluid; if this were achieved, it would reduce the duality, rather the many-sidedness, of physical phenomena to a simple unity of scheme; it would be the ultimate conceivable simplification. The celebrated vortex theory of matter makes the indestructible material atoms consist in vortex rings in a primordial fluid medium, structureless, homogeneous, and frictionless, and makes the forces between the atoms which form the groundwork of less fundamental theories consist in the actions excited by these vortices on one another through the inertia of the fluid which is their basis-actions which are instantaneously transmitted if the fluid is supposed to be absolutely incompressible.

In case this foundation proves insufficient, there is another idea of Lord Kelvin's by which it may be supplemented. The characteristic properties of radiation, which forms so prominent an element in actual phenomena, can be explained by the

1 A paper read before the Royal Society on December 7,1893 , by Dr. Joseph Larmor, F.R.S., Fellow of St. John's College, Cambridge. 
existence of an elastic medium for its transmission at a finite, though very great, speed; such a medium renders an excellent account of all its relations, if we assume it to possess inertia and to be endowed with some elastic quality of resistance to disturbance roughly analogous to what we can observe and study in ordinary elastic solids of the relatively incompressible kind, such as indiarubber and jellies. Lord Kelvin has been the promoter and developer of a view by which the elastic forces between parts of such a medium may be to some extent got rid of as ultimate elements, and be explained by the inertia of a spinning motion of a dynamically permanent kind, which is distributed throughout its volume. If we imagine very minute rapidly-spinning fly-wheels or gyrostats spread through the medium, they will retain their motion for ever, in the absence of friction on their axles, and they will thus form a concrete dynamical illustration of a type of elasticity which arises solely from inertia ; and this illustration will be of great use in realising some of the peculiarities of a related type, which I believe can be thoroughly established as the actual type of elasticity transmitting all radiations, whether luminous and thermal or electrical-for they are all one and the same-through the ultimate medium of fluid character of which the vortices constitute matter.

It has always been the great puzzle of theories of radiation how the medium which conveys it by transverse vibrations, such as we know directly only in media of the elastic-solid type, could yet be so yielding as to admit of the motion of the heavenly bodies through it absolutely without resistance. According to the view of the constitution of the æther which is developed in this paper, not only are these different properties absolutely consistent with each other, but it is, in fact, their absolute and rigorous coexistence which endows the medium with the qualities necessary for the explanation of a further very wide class of phenomena. The remark which is the key to this matter has been already thrown out by Lord Kelvin, in connection with Sir George Stokes's suggested explanation of the astronomical aberration of light. The motion of the ultimate homogeneous frictionless fluid medium, conditioned by the motion of the vortices existing in it, is, outside these vortices, of an absolutely irrotational character. Now, suppose the medium is endowed with elasticity of a purely rotational type, so that its elastic quality can be called into play only by absolute rotational displacement of the elements of the medium; just as motion of translation of a spinning gyrostat calls into play no reaction, while any alteration of the absolute position of its axis in space is resisted by an opposing couple. As regards the motion of the medium involved in the movements of its vortices, this rotational elasticity remains completely latent, as if it did not exist ; and we can at once set down the whole theory of the vortical hydrodynamical constitution of matter as a part of the manifestations of an ultimate medium of this kind.

The explanation of the laws of physical optics advanced by Fresnel, and verified by comparison with the phenomena which was possible in several very exact ways, chiefly by himself and Brewster, was, about the year 1835 , engaging the attention of several of the chief mathematicians of that time-Augusin Cauchy in France, Franz Neumann in Germany, George Green in England, and James MacCullagh in Ireland. The prevalent mode of attacking the problem was through the analogy with the propagation of elastic waves in solid bodies; and the comparison of Fresnel's laws of propagation in crystalline media with the results of the mathematical theory of the elasticity of crystalline bodies gave abundance of crucial tests for the verification, modification, or disproof of the principles assumed in these investigations.

The greatest achievement of MacCullagh is that contained in his menoir of 1839 , entitled an "Essay towards a Dynamical Theory of Crystalline Reflexion and Refraction." $\mathrm{He}$ is in quest of a dynamical foundation for the whole scheme of optical laws, which had been notably extended and confirmed by himself already. He recognises, I think for the first time in a capital physical problem, that what is required is the discovery of the potential-energy function of Lagrange on which the action of the medium depends, and that the explanation of the form of that function is another question which can be treated separately. His memoir is subsequent to, but apparently quite independent of, that of Green, in which Green restricted the medium to a constitution like an elastic solid, laid down the general laws of such constitution for the firs time, and made a magnificent failure of his attempt to explain optical phenomena on that basis. If this thing was to be done, the power, simplicity, and logical rigour of Green's analysis might have been expected to do it; and nothing further has come of the matter until the recent new departure of Lord Kelvin in his speculation as to a labile elastic-solid æther. To return to MacCullagh, he is easily able to hit off a simple form of the potential-energy function, which-on the basis of Lagrange's general dynamics, or more compactly on the basis of the law of Least Action-absolutely sweeps the whole field of optical theory so far as all phenomena are concerned in which absorption of the light does not play a prominent part. $\mathrm{He}$ is confident, as any one who follows him in detail must be, that he is on the right track. He tries hard to obtain a dyna mical basis for his energy-function, that is, to imagine some material medium that shall serve as a model for it, and illustrate its possibility and its mode of action; he records his failure in this respect, but at the same time he protests against the limited view which would tie down the unknown and in several ways mysterious and paradoxical properties of the luminiferous medium to be the same as those of an ordinary elastic solid.

The form of MacCullagh's energy -function was derived by him very easily from the consideration of the fact that it is required of it that it shall produce, in crystalline media, plane-polarised waves propagated by diiplacements in the plane of the wave front. Though he seems to put his reasoning as demonstrative on this point, it has been pointed out by Sir George Stokes, and is indeed obvious at once from Green's results, that other forms of the energy-function beside MacCullagh's woxld satisfy this condition. But the important point as regards MacCullagh's function is that it makes the energy in the medium depend solely on the absolute rotational displacements of its elements from their equilıbrium orientations, not at all on its distortion or compression, which are the quantities on which the elasticity of a solid would depend according to Green.

Starting from this conception of rotational elasticity, it can be shown that, if we neglect for the moment optical dispersion, every crystalline optical medium has three principal elastic axes, and its wave-surface is precisely that of Fresnel, while the laws of reflexion and refraction agree precisely with experiment. Further, it follows from the observed fact of transparency in combination with dispersion, that the dispersion of a wave of permanent type is properly accounted for by the addition to the equations, therefore to the energy-function, of subsidiary terms involving spacial differentiations of higher order. To preserve the medium hydrodynamically a perfect fluid, these terms also must satisfy the condition that the elasticity of the medium is thoroughly independent of compression and distortion of its elements, and wholly dependent on absolute rotation. It can be shown, I believe, that this restriction limits the terms to two kinds, one of which retains Fresnel's wave-surface unaltered, while the other modifies it in a definite manner stated without proof by MacCullagh; but the first terms depend on an interaction between the dispersive property and the wave motion itself, while the second terms involve the square of the dispersive quality. It seems clear that the second type involves only phenomena of a higher order of small quantities than we are here considering; thus an account of dispersion remains which retairs Fresnel's wave-surface unaltered for each homogeneous constituent of the light, while it includes the dispersion of the optic axes in crystals both as regards their magnitudes and directions-results quite unapproached by any other theory ever entertained.

In this analysis of dispersions, all terms have been omitted which possess a unilateral character, such as would be indicated in actuality by rotatory polarisation and other like phenomena. The laws of crystalline material structures seem to prohibit the occurrence of such asymmetry as these terms would indicate, except to the very small extent evidenced by the hemihedral faces of quartz crystals. The influence of this asymmetric arrangement of the molecules on the optical medium must be very much smaller still, for the rotatory terms are in all media exceedingly minute compared with the ordinary dispersional terms. The form of these rotatory terms in the energy-function is at once definitely assigned by our condition of perfect fluidity of the medium, both for crystals and for rotational liquids such as turpentine, and this form is the one usually accepted, on MacCullagh's suggestion, as yielding a correct account of the phenomena.

When dispersional terms are included in the energy function,

NO. 1263 , VOL. 49] 
our continuous analysis is not any longer applicable to the problem of reflexion; the conditions at the interface are altogether too numerous to be satisfied by the available variables. There is in fact discontinuity at the interface in the discrete molecular structure, such as could not be representable by a continuous analysis. But if we proceed by the method of rays, and assume that there is a play of surface forces which do not absorb any energy, while they adjust the dispersional part of the stress, it appears that reflexion is independent of dispersion.

The problem of the æther has been first determinedly attacked from the side of electrical phenomena by Clerk Maxwell in quite recent times ; his great memoir on a "Dynamical Theory of the Electromagnetic Field " is of date r864. It is in fact only comparatively recently that the observation of Oersted, and the discoveries and deductions of Ampère, Faraday, and Thomson had accumulated sufficient material to allow the question to be profitably attacked from this side. Even as it is, our notions of what constitute electric and magnetic phenomena are of the vaguest as compared with our ideas of what constitutes radiation, so that Maxwell's views involve difficulties, not to say contradictions, and in places present obstacles which are to be surmounted, not by logical argument or any clear representation, but by the physical intuition of a mind saturated with this aspect of the phenomena. Many of these obstacles may, I think, be removed by beginning at the other end, by explaining electric actions on the basis of a mechanical theory of radiation, instead of radiation on the basis of electric actions. The strong point of Maxwell's theory is the electromotive part, which gives an account of electric radiation and of the phenomena of electromagnetic induction in fixed conductors; and this is in keeping with the remark just made. The nature of electric displacement, of electric and magnetic forces on matter, of what Maxwell calls the electrostatic and the magnetic stress in the medium, of electrochemical phenomena, are all left obscure.

We shall plunge into the subject at once from the optical side, if we assume that dielectric polarisation consists in a strain in the æther, of the rotational character contemplated above The conditions of internal equilibrium of a medium so strained are easily worked out from MacCullagh's expression for W, its potential energy. If the vector $(f, g, h)$ denote the curl or vorticity of the actúal linear displacement of the medium, or twice the absolute rotation of the portion of the medium at the point considered, and the medium is supposed of crystalline quality and referred to its principal axes, so that

$$
\mathrm{W}=\frac{1}{2} \int\left(a^{2} f^{2}+b^{2} g^{2}+c^{2} h^{2}\right) d \tau,
$$

where $d \tau$ is an element of volume, it follows easily that for internal equilibrium we must have

$$
a^{2} f d x+b^{2} g d y+c^{2} h d z=-d \mathrm{~V},
$$

a complete differential, and that over any boundary enclosing a region devoid of elasticity the value of $\mathrm{V}$ must be constant. Such a boundary is the surface of a conductor; $\mathrm{V}$ is the electric potential in the field due to charges on the conductors; $(f, g, h)$ is the electric displacement in the field, circuital by its very nature as a rotation, and $\left(a^{2} f, b^{2} g, c^{2} h\right)$ is the electric force derived from the electric potential $V$. The charge on a conductor is the integral of $(f, g, h)$ over any surface enclosing it, and cannot be altered except by opening up a channel devoid of elasticity, in the medium, between this conductor and some other one; in other words, electric discharge can take place only by rupture of the elastic quality of the æthereal medium.

At the interface between two dielectric media, taken to be crystalline as above, the condition comes out to be that the tangential electric force is continuous. When the circumstances are those of equilibrium, and therefore an electric potential may be introduced, this condition allows discontinuity in the value of the potential in crossing the interface, but demands that the amount of this discontinuity shall be the same all along the interface; these are precisely the circumstances of the observed phenomena of voltaic potential differences. The component, normal to the interface, of the electric displacement is of course always continuous, from the nature of that vector as a flux.

It may present itself as a difficulty in this theory that, as the electric displacement is the rotational displacement of the medium, its surface integral over any sheet should be equal to the line integral of the linear displacement of the medium round the edge of the sheet; therefore that for a closed sheet surrounding a conductor this integral should be null, which would involve the consequence that the electric charge on a conductor cannot be different from null. This line of argument, however, implies that the linear displacement is a perfectly continuous one, which is concomitant with and required by the electric displacement. The legitimate inference is that the electric displacement in the medium which corresponds to an actual charge cannot be set up without some kind of discontinuity or slip in the linear displacement of the medium ; in other words, that a conductor cannot receive an electric charge without rupture of the surrounding medium; nor can it lose a charge once received without a similar rupture. The part of the linear displacement that remains, after this slip or rupture has been deducted from it, is of elastic origin, and must satisfy the equations of equilibrium of the medium.

We can produce in imagination a steady electric current, without introducing the complication of galvanic batteries, in the following manner, and thus examine in detail all that is involved, on the present theory, in the notion of a current. Suppose we have two charged condensers, with one pair of coatings connected by a narrow conducting channel, and the other pair connected by another such channel, as in the annexed diagram, where the dark regions are dielectric

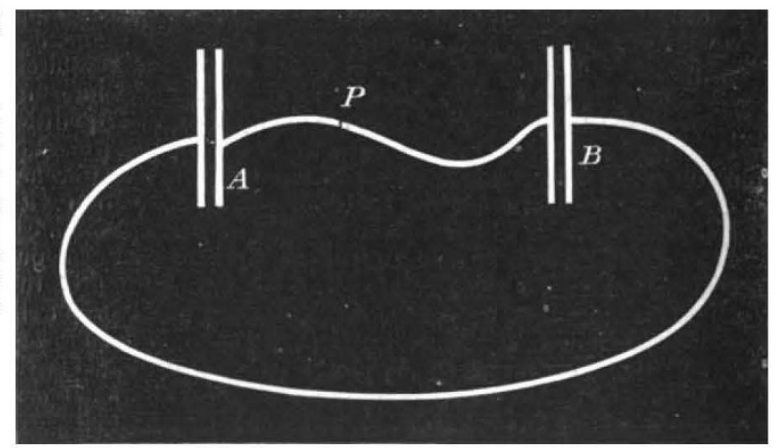

and the white regions conducting. If we steadily move towards each other the two plates of the condenser A, a current will flow round the circuit, in the form of a conduction current in the conductors and a displacement current across the dielectric plates of the condensers. Let us suppose the thicknesses of these dielectric plates to be excessively small, so as to minimise the importance of the displacement part of the current. There is then practically no electric force and therefore no electric displacement in the surrounding dielectric field, except between the plates of the condensers and close to the conducting wires. Consider a closed surface passing between the faces of the condenser $A$, and intersecting the wire at a place P. A movement of the faces of this condenser alters the electric force between them, and therefore alters the electric displacement across the portion of this closed surface which lies in that part of the field; as we have seen, there is practically no displacement anywhere else in the field except at the conducting wire; therefore to preserve the law of the circuital character of displacement throughout the whole space, we must suppose that this alteration is compensated by a very intense change of displacement at the conducting wire. So long as the movement of the plates continues, as long does this flow of displacement along the wire go on; it constitutes the electric current in the wire. Now, in calculating the magnetic force in the field, which is the velocily of the æthereal medium; from the change of electric displacement, we must include in our integration the effect of this sheet of electric displacement flowing along the surface of the perfectly conducting wires, for exactly the same reason as in the correlative problem in hydrodynamics, of calculating the velocity of the fluid from the distribution of vorticity in it, Helmholtz had to consider a vortex sheet as existing over each surface across which the motion is discontinuous.

(To be continued.) 